\title{
Efficient approach to the free energy of crystals via Monte Carlo simulations
}

\author{
G. Navascués* \\ Departamento de Física Teórica de la Materia Condensada and Instituto de Física de la Materia Condensada (IFIMAC), \\ Universidad Autónoma de Madrid, E-28049, Madrid, Spain \\ E. Velasco ${ }^{\dagger}$ \\ Departamento de Física Teórica de la Materia Condensada, Instituto de Física de la Materia Condensada (IFIMAC) \\ and Instituto de Ciencia de Materiales Nicolás Cabrera, Universidad Autónoma de Madrid, E-28049, Madrid, Spain
}

(Received 30 April 2015; published 4 August 2015)

\begin{abstract}
We present a general approach to compute the absolute free energy of a system of particles with constrained center of mass based on the Monte Carlo thermodynamic coupling integral method. The version of the FrenkelLadd approach [J. Chem. Phys. 81, 3188 (1984)], which uses a harmonic coupling potential, is recovered. Also, we propose a different choice, based on one-particle square-well coupling potentials, which is much simpler, more accurate, and free from some of the difficulties of the Frenkel-Ladd method. We apply our approach to hard spheres and compare with the standard harmonic method.
\end{abstract}

DOI: 10.1103/PhysRevE.92.022103

PACS number(s): 05.70.Fh, 64.10.+h

\section{INTRODUCTION}

One of the most popular methods to obtain the Helmholtz free energy of a crystalline solid by means of computer simulation is due to Frenkel and Ladd (FL) [1,2]. This method has been extensively used and applied to many different interaction models $[3,4]$. The method essentially consists of three steps: (i) The system of particles is coupled to an Einstein crystal (EC), such that each particle is subject to a strong, localizing harmonic potential with force constant $k_{\mathrm{EC}}$; the free energy of the Einstein crystal (plus corrections), $F_{\mathrm{EC}}^{(\mathrm{CM})}$, where "CM" denotes the center of mass, is supposed to be known. (ii) The system is slowly decoupled from the Einstein crystal (i.e., $k_{\mathrm{EC}}$ is slowly reduced to zero), and the free energy change $\Delta F_{\mathrm{EC}}^{(\mathrm{CM})}$ involved in this process is obtained by thermodynamic integration with respect to $k_{\mathrm{EC}}$. Computer simulation is used to evaluate the appropriate integrand. In these two steps the CM is constrained to be fixed at the center of the simulation box. (iii) The CM constraint is removed and the free energy conveniently corrected, with a contribution $\Delta F$. The free energy is finally obtained as

$$
F=F_{\mathrm{EC}}^{(\mathrm{CM})}+\Delta F_{\mathrm{EC}}^{(\mathrm{CM})}+\Delta F=F^{(\mathrm{CM})}+\Delta F .
$$

where $F^{(\mathrm{CM})}$ is the Helmholtz free energy with fixed CM. Details of the method can be found elsewhere [1,2].

The practical implementation of the Frenkel-Ladd method contains some subtleties. Recently [5], we have addressed a question associated with step (iii) and the problem of how to evaluate the free-energy contribution $\Delta F$ incurred when the CM constraint is lifted. We demonstrated that $\Delta F$ depends not only on density (as previously supposed) but also on dimensionality, box shape, and system size. The general analytic dependence of $\Delta F$ with system size, in particular, is not known at present, which hampers a rigorous estimation of the thermodynamic limit. In general, the free-energy

\footnotetext{
*guillermo.navascues@uam.es

†enrique.velasco@uam.es
}

correction per particle is small and quickly tends to zero in the thermodynamic limit. As a consequence, it might be more sensible to estimate free energies of crystals with fixed centers of mass, i.e., to look for the free energy at the thermodynamic limit, putting aside those contributions which go to zero at that limit. Of course, when interested in size effects, one should take into account all (unknown) terms, though probably only for very small systems would the CM correction $\Delta F$ give a significant contribution (see Ref. [5]).

In this paper we address two issues. The first is the derivation of a general theoretical framework to obtain the Helmholtz free energy $F^{(\mathrm{CM})}$ via Monte Carlo simulation; the FL method results as a particular case. The second addresses some technical limitations of the FL method which are largely avoided by using another choice for coupling potential, namely the square-well model. In particular, our approach provides the exact free energy of the reference system, step (i) above, and simplifies the thermodynamic integration of step (ii). Our approach is in practice more accurate than the FL method, which does not provide a systematic route when high accuracy is required.

The paper is arranged as follows. In Sec. II we compile all the basic equations needed to obtain a general derivation of the partition function of the reference system, the details being relegated to Appendix. Section III is devoted to a new proposal for coupling potential, namely the square-well model, and the advantages of this choice are discussed. To illustrate the method, we apply it to hard spheres in Sec. IV, where a critical comparison of the procedure with the FL method is made. In the final section, we conclude with a summary of the aims and results of the paper.

\section{GENERAL RESULTS}

The CM constraint only concerns spatial coordinates. Therefore, in order to simplify the algebra, we consider spherical particles (the generalization to asymmetric particles is trivial and will done elsewhere). Consider a system of $N$ identical classical particles, each of mass $m$, at temperature $T$, 
in a volume $V$, under the constraint of fixed CM (spatial and momentum) coordinates $\boldsymbol{R}$ and $\boldsymbol{P}$. The corresponding free energy can be written as:

$$
F^{(\mathrm{CM})}(\boldsymbol{R}, \boldsymbol{P})=-k T \ln \left[\Pi^{(\mathrm{CM})}(\boldsymbol{P}) Z^{(\mathrm{CM})}(\boldsymbol{R})\right] .
$$

The kinetic partition function can be expressed analytically as [5]

$$
\Pi^{(\mathrm{CM})}(\boldsymbol{P})=\frac{\Lambda_{\mathrm{CM}}^{3}}{\Lambda^{3 N}} e^{-\beta T_{\mathrm{CM}}(\boldsymbol{P})},
$$

with $\Lambda=\left(h^{2} / 2 \pi m k T\right)^{1 / 2}$ the usual thermal wavelength, $\Lambda_{\mathrm{CM}}=\left(h^{2} / 2 \pi N m k T\right)^{1 / 2}$ the CM thermal wavelength, and $T_{\mathrm{CM}}=P^{2} / 2 N m$ the CM kinetic energy. $k$ and $h$ are Boltzmann and Planck constants, respectively. With the restriction on the localization of the $\mathrm{CM}$, the configuration partition function is

$Z^{(\mathrm{CM})}(\boldsymbol{R})=\int_{V} d \boldsymbol{r}_{1} \ldots \int_{V} d \boldsymbol{r}_{N} \delta\left(\boldsymbol{R}-\frac{1}{N} \sum_{i=1}^{N} \boldsymbol{r}_{i}\right) e^{-\beta U\left(\boldsymbol{r}_{1}, \ldots, \boldsymbol{r}_{N}\right)}$

where

$$
U\left(\boldsymbol{r}_{1}, \ldots, \boldsymbol{r}_{N}\right)=\sum_{i=1}^{N} \sum_{i<j} \varphi\left(r_{i j}\right)
$$

is the energy of the system assuming a pairwise interaction potential $\varphi(r)$ and $\beta \equiv 1 / k T$. In order to evaluate the contribution of the configuration partition function to the free energy, namely $F^{(\mathrm{CM})}(\boldsymbol{R})=-k T \ln Z^{(\mathrm{CM})}(\boldsymbol{R})$, the system is coupled, through a thermodynamic integral, to a reference system whose free energy is known. To this end let us consider a crystalline phase with lattice vectors $\left\{\boldsymbol{R}_{i}\right\}$. We couple the system to a one-particle external potential with the same crystal structure, $\sum_{i=1}^{N} \lambda V\left(\boldsymbol{r}_{i}-\boldsymbol{R}_{i}\right) \equiv \sum_{i=1}^{N} \lambda V_{i}$. Each one-particle potential, $\lambda V_{i}$, acts on the corresponding particle by enhancing its localization about its equilibrium lattice site $\boldsymbol{R}_{i}$. Its strength, $\lambda$, plays the role of a coupling parameter. Let the interaction between two particles be modified as

$$
\varphi_{\lambda}(r)=\varphi_{\mathrm{ref}}(r)+\left(1-\frac{\lambda}{\lambda^{*}}\right)\left[\varphi(r)-\varphi_{\mathrm{ref}}(r)\right],
$$

where $0 \leqslant \lambda \leqslant \lambda^{*}$, which gives the total energy

$$
U_{\lambda}=U_{\mathrm{ref}}+\left(1-\frac{\lambda}{\lambda^{*}}\right)\left(U-U_{\mathrm{ref}}\right)
$$

with

$$
U_{\mathrm{ref}}=\sum_{i=1}^{N} \sum_{i<j} \varphi_{\mathrm{ref}}\left(r_{i j}\right)
$$

where $\varphi_{\text {ref }}(r)$ is an appropriate reference pairwise potential and $\lambda^{*}$ is an upper value for the coupling parameter. Note that, on introducing the one-particle external potential attached to particular lattice sites, particles become distinguishable. Of course, the kinetic factor is still given by Eq. (3), while the configuration part now depends on $\lambda$ :

$$
\begin{aligned}
Z^{(\mathrm{CM})}(\boldsymbol{R} ; \lambda)= & \int_{V} d \boldsymbol{r}_{1} \ldots \int_{V} d \boldsymbol{r}_{N} \delta\left(\boldsymbol{R}-\frac{1}{N} \sum_{i=1}^{N} \boldsymbol{r}_{i}\right) \\
& \times e^{-\beta\left[\sum_{i=1}^{N} \lambda V_{i}+U_{\mathrm{ref}}+\left(1-\frac{\lambda}{\lambda^{*}}\right)\left(U-U_{\mathrm{ref}}\right)\right]} .
\end{aligned}
$$

In the limit $\lambda=0$ the partition function of the original system is recovered, except that it corresponds to a system of distinguishable particles, while for $\lambda=\lambda^{*}$ one obtains the reference-system partition function:

$$
\begin{aligned}
Z^{(\mathrm{CM})}\left(\boldsymbol{R} ; \lambda^{*}\right)= & Z_{\mathrm{ref}}^{(\mathrm{CM})}(\boldsymbol{R}) \\
= & \int_{V} d \boldsymbol{r}_{1} \ldots \int_{V} d \boldsymbol{r}_{N} \delta\left(\boldsymbol{R}-\frac{1}{N} \sum_{i=1}^{N} \boldsymbol{r}_{i}\right) \\
& \times e^{-\beta\left(\sum_{i=1}^{N} \lambda^{*} V_{i}+U_{\mathrm{ref}}\right)} .
\end{aligned}
$$

The standard coupling formalism [6] allows us to express the free-energy difference of a system with different coupling parameters as

$$
F\left(\lambda_{2}\right)-F\left(\lambda_{1}\right)=\int_{\lambda_{1}}^{\lambda_{2}} d \lambda\left\langle\frac{\partial F}{\partial \lambda}\right\rangle_{\lambda}
$$

where $\langle\ldots\rangle_{\lambda}$ denotes an ensemble average over a system with coupling parameter $\lambda$, obtained from simulation. In our problem we take $\lambda_{2}=0$ and $\lambda_{1}=\lambda^{*}$, so Eq. (11) is easily seen to transform to

$$
\begin{aligned}
F^{(\mathrm{CM})}(\boldsymbol{R} ; \lambda=0) \equiv & -k T \ln Z_{\mathrm{ref}}^{(\mathrm{CM})}(\boldsymbol{R}) \\
& +\int_{\lambda^{*}}^{0} d \lambda\left\langle\sum_{i=1}^{N} V_{i}-\frac{U-U_{\mathrm{ref}}}{\lambda^{*}}\right\rangle_{\lambda},
\end{aligned}
$$

We note that the linear coupling procedure described here, with a single coupling constant, is the simplest one. The extension to more elaborate couplings, implying additional coupling parameters and more complex simulations, can be done without any difficulty. Note also that, for linear couplings, there are different but equivalent choices in the literature to define the coupling; for instance, sometimes $\lambda$ is redefined to $1-\lambda$ or the limit values 0 and $\lambda^{*}$ are changed to 0 and 1 , and so on.

The above results are well known. The reference system invariably used in the literature is the Einstein crystal, i.e., the harmonic-coupling method, which gives rise to the FL method. Here we obtain a simple integral expression, valid for any coupling potential, which allows us to identify the most appropriate coupling potential in each problem; in fact, we will see that, for general purposes, the square-well potential is simpler to use and numerically more accurate than the harmonic potential. Of course, the general free-energy expression reduces to the FL expression when a harmonic potential is used.

The partition function of the reference system, Eq. (10), can be formally written as:

$$
Z_{\mathrm{ref}}^{(\mathrm{CM})}(\boldsymbol{R}) \equiv Z_{\mathrm{ref}}^{(\mathrm{CM}, 0)}(\boldsymbol{R})\left\langle\left\langle e^{-\beta U_{\mathrm{ref}}}\right\rangle\right\rangle_{\lambda^{*}}
$$


where

$$
Z_{\mathrm{ref}}^{(\mathrm{CM}, 0)}(\boldsymbol{R})=\int_{V} d \boldsymbol{r}_{1} \cdots \int_{V} d \boldsymbol{r}_{N} \delta\left(\boldsymbol{R}-\frac{1}{N} \sum_{i=1}^{N} \boldsymbol{r}_{i}\right) e^{-\beta \sum_{i=1}^{N} \lambda^{*} V_{i}}
$$

is a noninteracting partition function and $\langle\langle\cdots\rangle\rangle_{\lambda^{*}}$ stands for an ensemble average over configurations of noninteracting particles. This average can be evaluated by simulation, or one can try to develop some approximation. We see below that if one chooses the appropriate coupling potential, this factor is exactly equal to 1 , and any explicit evaluation is unnecessary. $Z_{\mathrm{ref}}^{(\mathrm{CM}, 0)}(\boldsymbol{R})$ is formally equivalent to an elementary weighted random walk; Appendix contains all the mathematical details (which are otherwise irrelevant for our proposes; here we only take advantage of the results). In practical cases, the CM is located at the geometric center of the system, $\boldsymbol{R}=\boldsymbol{R}_{\mathrm{GC}}$, and the coupling potential depends only on the distance from the particle equilibrium sites (e.g., harmonic potential in the FL method). In fact, all workers have implicitly taken $\boldsymbol{R}=\boldsymbol{R}_{\mathrm{GC}}$, too. In this case Eq. (A16) of Appendix yields the general expression

$$
Z_{\mathrm{ref}}^{(\mathrm{CM}, 0)}=V_{\mathrm{eff}}^{N} N^{3 / 2} e^{-\beta \lambda^{*} V_{0}}\left[\frac{8}{3} \pi^{2} \int d r r^{4} \omega(r)\right]^{-3 / 2},
$$

where

$$
V_{\mathrm{eff}} \equiv \int_{V} d \boldsymbol{r} e^{-\beta \lambda^{*}\left[V(r)-V_{0}\right]}
$$

is the effective volume explored by each noninteracting particle, weighted by the Boltzmann exponential, and

$$
\omega(r) \equiv e^{-\beta \lambda^{*}\left[V(r)-V_{0}\right]} / V_{\text {eff }}
$$

is the probability density to visit the distance $r$ and $V_{0}$ the minimum of $V$. Explicit definitions for the quantities $V_{\text {eff }}$ and $\omega$ are useful only in the context of Appendix, and here we just regard Eq. (15) as a simple analytic result.

\section{THEORETICAL EXPRESSION BASED ON A SQUARE-WELL COUPLING}

\section{A. Reference system}

We introduce a new approach that uses the square-well (SW) model as a coupling potential. The model has two parameters, the well radius $R_{\mathrm{SW}}$ and depth $\epsilon$, both of which can be used as coupling parameters. Here we will fix $R_{\mathrm{SW}}$ and use $\epsilon$ as a coupling parameter. The coupling potential on an arbitrary particle is taken as

$$
\lambda V \equiv \epsilon \Theta\left(r-R_{\mathrm{SW}}\right),
$$

where $\Theta(r)$ is the step function. In order for a particle to be inside the SW potential, it has to satisfy the condition $r<R_{\mathrm{SW}}$.

The reference system is defined as follows: each particle is under the effect of a infinitely deep SW potential, but we locate the zero energy level in the well bottom $V_{0}=0$, i.e., in Eq. (18) $\epsilon$ is zero inside the well and infinite outside the well. In this way we simplify the algebra by avoiding a trivial factor in the partition function, namely that associated to the energy of the reference system, which would be canceled out at the end of the decoupling proccess. The square wells are localized at the lattice sites corresponding to the actual system. Besides, particles interact with each other through a finiterange reference pairwise potential, $\varphi_{\text {ref }}$, such that particles located at different wells do not see each other. Therefore the reference system is strictly noninteracting and, consequently, $\left\langle\left\langle e^{-\beta U_{\text {ref }}}\right\rangle\right\rangle_{\epsilon=\infty} \equiv 1$. In this case $V_{\text {eff }}$ is the volume of the square well (i.e., that of a sphere of radius $R_{\mathrm{SW}}$ ), the integral in Eq. (15) is trivial, and the reference partition function is given by

$$
Z_{\mathrm{ref}}^{(\mathrm{CM})}=\left(\frac{4 \pi R_{\mathrm{SW}}^{3}}{3}\right)^{N} N^{3 / 2}\left(\frac{5}{2 \pi R_{\mathrm{SW}}^{2}}\right)^{3 / 2} .
$$

It is easy to realize that the determination of $R_{\mathrm{SW}}$ is a simple geometrical problem and, as shown below, a trivial argument provides the optimal values for this parameter.

\section{B. Thermodynamic integrals}

We now show that the first integrand in Eq. (12) is simply the probability of finding a particle outside of its square well. We have:

$$
\int_{\lambda_{1}}^{\lambda_{2}} d \lambda\left\langle\sum_{i=1}^{N} V_{i}\right\rangle_{\lambda}=\int_{\epsilon_{1}}^{\epsilon_{2}} d \epsilon\left\langle\sum_{i=1}^{N} \Theta\left(r_{i}-R_{\mathrm{SW}}\right)\right\rangle_{\epsilon} .
$$

Note that

$$
\Theta\left(r_{i}-R_{\mathrm{SW}}\right)=n_{i},
$$

where $n_{i}$ is equal to 1 if the particle is outside of the well and 0 otherwise. Now let $\langle n\rangle_{\epsilon}$ be the mean number of particles outside of their well, i.e.,

$$
\langle n\rangle_{\epsilon}=\frac{1}{N} \sum_{i=1}^{N}\left\langle n_{i}\right\rangle_{\epsilon} .
$$

Note that $0 \leqslant\langle n\rangle_{\epsilon} \leqslant 1$. Then the coupling integral can be written as

$$
\int_{\lambda_{1}}^{\lambda_{2}} d \lambda\left\langle\sum_{i=1}^{N} V_{i}\right\rangle_{\lambda}=N \int_{\epsilon_{1}}^{\epsilon_{2}} d \epsilon\langle n\rangle_{\epsilon} .
$$

In a computer simulation $\langle n\rangle_{\epsilon}$ is obtained by simply counting particles.

A useful observation is that, for well depths beyond some large value, $\epsilon>\epsilon^{*}$, the Boltzmann exponential is dominated by the coupling potential, regardless of the interactions $U$ and $U_{\text {ref }}$. This observation suggests realizing the coupling to the square well in two steps. In the first, $\epsilon$ is decreased from infinity to $\epsilon^{*}$, setting the interaction between particles to be

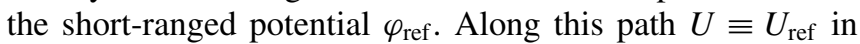
Eq. (12) [or Eq. (9)]. The total coupling integral associated to this step then reduces to

$$
I_{1}=N \int_{\infty}^{\epsilon^{*}} d \epsilon\langle n\rangle_{\epsilon} .
$$

Also the probability to find a particle outside its well is given to a high accuracy by the Boltzmann factor of the SW potential, i.e., $\langle n\rangle_{\epsilon} \simeq \exp (-\epsilon / k T)$, and the thermodynamic integral $I_{1}$ can be carried out exactly to give

$$
I_{1}=-N k T\langle n\rangle_{\epsilon^{*}},
$$


where $\langle n\rangle_{\epsilon^{*}}$ will be obtained as a subproduct in a second coupling step. In the second step we decouple the SW potential $\epsilon^{*} \Theta\left(r-R_{\mathrm{SW}}\right)$ and, at the same time, the interaction potential $\varphi-\varphi_{\text {ref }}$ is coupled; in this way the actual system is obtained. The corresponding coupling integral, Eq. (12), reads:

$$
I_{2}=N \int_{\epsilon^{*}}^{0} d \epsilon\left[\langle n\rangle_{\epsilon}-\frac{1}{N \epsilon^{*}}\left\langle U-U_{\text {ref }}\right\rangle_{\epsilon}\right] .
$$

Note that the first integrand at the lower integral limit is just $\langle n\rangle_{\epsilon^{*}}$, which means that a single thermodynamic simulation with a single coupling parameter, associated with the second step, is all that is needed to obtain the total thermodynamic integral $I_{1}+I_{2}$.

\section{Comparison between SW and harmonic couplings}

Evaluation of (12) using Eqs. (19), (25), and (26) leads to an expression for $F_{\text {conf }}^{(\mathrm{CM})}$ :

$$
\begin{aligned}
\frac{\beta F_{\mathrm{conf}}^{(\mathrm{CM})}}{N}= & -\frac{1}{N} \ln \left[\left(\frac{4 \pi R_{\mathrm{SW}}^{3}}{3}\right)^{N} N^{3 / 2}\left(\frac{5}{2 \pi R_{\mathrm{SW}}^{2}}\right)^{3 / 2}\right] \\
& +\beta \int_{\epsilon^{*}}^{0} d \epsilon\left[\langle n\rangle_{\epsilon}-\frac{1}{N \epsilon^{*}}\left\langle U-U_{\mathrm{ref}}\right\rangle_{\epsilon}\right]-\langle n\rangle_{\epsilon^{*}}
\end{aligned}
$$

Proceeding in the same way, but using the harmonic coupling potential

$$
\lambda V_{i} \equiv \frac{1}{2} k_{\mathrm{EC}} r_{i}^{2},
$$

where the coupling parameter $k_{\mathrm{EC}}$ is the force constant of the harmonic potential, we recover the FL expression:

$$
\begin{aligned}
\frac{\beta F_{\mathrm{conf}}^{(\mathrm{CM})}}{N}= & -\frac{1}{N} \ln \left[\left(\frac{2 \pi}{\beta k_{\mathrm{EC}}^{*}}\right)^{3 N / 2} N^{3 / 2}\left(\frac{\beta k_{\mathrm{EC}}^{*}}{2 \pi}\right)^{3 / 2}\right] \\
& +\frac{\beta}{2} \int_{k_{\mathrm{EC}}^{*}}^{0} d k_{\mathrm{EC}}\left[\left\langle r^{2}\right\rangle_{k_{\mathrm{EC}}}-\frac{2}{N k_{\mathrm{EC}}^{*}}\left\langle U-U_{\mathrm{ref}}\right\rangle_{k_{\mathrm{EC}}}\right] \\
& -\frac{1}{N} \ln \left\langle\left\langle\exp \left\{-\beta U_{\mathrm{ref}}\right\}\right\rangle\right\rangle_{k_{\mathrm{EC}}^{*}}
\end{aligned}
$$

where $k_{\mathrm{EC}}^{*}$ is the force constant of the reference system.

There is a subtle question with the first term in Eq. (29), since it is an approximation to the exact result. Strictly speaking, obtaining an exact analytic expression would involve assuming that the system is infinite in extension so Eqs. (15) and (16) can be calculated. The accuracy of the term in (29), which is exact in the limit $k_{\mathrm{EC}}^{*}=\infty$, has not been estimated, but one would expect it to be very good for sufficiently large $k_{\mathrm{EC}}^{*}$. However, in the strict limit, the reference free energy diverges logarithmically. In contrast, for a SW coupling, the reference free energy is exact and finite and saturates quite rapidly to a constant value. Another difficulty with the harmonic coupling is that the last term of Eq. (29) needs an extra simulation or some kind of approximation (for example, Frenkel and Ladd derived a low-order virial approximation for hard spheres [1]).

Therefore, despite the formal similarity of the two methods, there are substantial practical differences which will become clearer in the illustrative example shown in the following section.

\section{APPLICATION TO HARD SPHERES (HS)}

In this section we show how to apply the SW coupling method. As will become apparent, the methodology does not depend on whether the interaction potential is continuous, discontinuous, or anisotropic. The numerical values given below are meant to illustrate the method and to give a critical comparison with the FL method. All energies are given in $k T$ units.

For a hard-sphere system (and, in fact, for any other hardbody system), the obvious reference interaction potential is the hard-sphere potential itself:

$$
\varphi_{\mathrm{ref}}(r) \equiv \varphi_{\mathrm{HS}}(r)= \begin{cases}\infty, & r<1 \\ 0, & r>1\end{cases}
$$

(distances are in HS diameter units). Because of the noninteraction condition in the reference system $(\epsilon=\infty)$, the radius $R_{\mathrm{SW}}$ must be $\leqslant(d-1) / 2$, the parameter $d$ being the nearestneighbor distance. From Eq. (30) we have $U=U_{\text {ref }} \equiv U_{\mathrm{HS}}$. Equation (27) then reduces to

$$
\begin{aligned}
\frac{\beta F_{\mathrm{conf}}^{(\mathrm{CM})}}{N}= & -\frac{1}{N} \ln \left[\left(\frac{4 \pi R_{\mathrm{SW}}^{3}}{3}\right)^{N} N^{3 / 2}\left(\frac{5}{2 \pi R_{\mathrm{SW}}^{2}}\right)^{3 / 2}\right] \\
& +\beta \int_{\epsilon^{*}}^{0} d \epsilon\langle n\rangle_{\epsilon}-\langle n\rangle_{\epsilon^{*}}
\end{aligned}
$$

Table I collects the results for various choices of density and $R_{\mathrm{SW}}$ (all MC simulations in this section where performed on a system of $N=4000$ spheres, averaging over $5 \times 10^{4} \mathrm{MC}$ steps; the error in averaged quantities was estimated to be $\sim 0.1 \%$ ).

The value of $R_{\mathrm{SW}}$ should not be too small to avoid bad statistics. See, e.g., the case $\rho=1.05 \sigma^{-3}$ in the table and the convergence of $\beta F_{\text {conf }}^{(\mathrm{CM})} / N$ with $R_{\mathrm{SW}}$. The choice $R_{\mathrm{SW}}=(d-1) / 2$ (the largest possible value) has the following advantages. For any sensible $R_{\mathrm{SW}}$ the reference free energy is positive and decreases with this parameter, while the two other (coupling) terms are negative. To obtain an optimal numerical difference, both contributions should be the smallest possible and therefore $R_{\mathrm{SW}}$ should be maximum. Also, with this choice, the largest contribution comes from the exact reference contribution. The coupling parameter $\epsilon^{*}$ is chosen such that its Boltzmann factor controls the system configurations (see previous section); it turns out to be of the order of a few $k T$, as could be expected. Note the sum of the two coupling terms is invariant with respect to $\epsilon^{*}$. Finally, note that the accuracy attained in $\beta F_{\text {conf }}^{(\mathrm{CM})} / N$ depends only on the simulation statistics and the integration method (quadrature or otherwise) used to evaluate the thermodynamic integral, second term in Eq. (31). The error introduced by the last term should be approximately the error in $\langle n\rangle_{\epsilon^{*}}$ times $\exp \left(-\beta \epsilon^{*}\right)$, which is completely negligible compared with the error of the thermodynamic integral, second term in Eq. (31).

Figure 1 illustrates the behavior of $\langle n\rangle$ as a function of $\beta \epsilon$, for several values of $R_{\mathrm{SW}}$, at reduced density $\rho \sigma^{3}=1.05$. Note that $\langle n\rangle$ is a smooth and short-ranged function, which becomes exponential already for small values of $\beta \epsilon$. As $R_{\mathrm{SW}}$ decreases the exponential behavior appears at larger values of $\beta \epsilon$ (this is another reason to favor large values of $R_{\mathrm{SW}}$ ). The effect of density on $\langle n\rangle$ is relative small, as can be seen in Fig. 2, which 
TABLE I. Contributions to the configurational free energy $\beta F_{\text {conf }}^{(\mathrm{CM})} / N$, see Eq. (12). Data obtained from Monte Carlo simulations of $N=4000$ hard spheres arranged in a FCC structure. Lengths are in units of the HS diameter.

\begin{tabular}{|c|c|c|c|c|c|c|c|c|c|}
\hline \multirow[b]{2}{*}{$R_{\mathrm{SW}}$} & \multicolumn{4}{|c|}{$\rho=1.05$ and $\epsilon^{*}=8 k T$} & \multirow[b]{2}{*}{$\epsilon^{*} / k T$} & \multicolumn{4}{|c|}{$\rho=1.05$ and $R_{\mathrm{SW}}=0.05218$} \\
\hline & $\beta F_{\text {ref }}^{(\mathrm{CM})} / N$ & $\beta \int_{\epsilon^{*}}^{0} d \epsilon\langle n\rangle_{\epsilon}$ & $-\langle n\rangle_{\epsilon^{*}}$ & $\beta F_{\mathrm{conf}}^{(\mathrm{CM})} / N$ & & $\beta F_{\text {ref }}^{(\mathrm{CM})} / N$ & $\beta \int_{\epsilon^{*}}^{0} d \epsilon\langle n\rangle$ & $-\langle n\rangle_{\epsilon^{*}}$ & $\beta F_{\text {conf }}^{(\mathrm{CM})} / N$ \\
\hline 0.00522 & 14.328 & -5.395 & -0.477 & 8.456 & 6 & 7.422 & -2.343 & -0.022 & 5.056 \\
\hline 0.01304 & 11.580 & -6.308 & -0.187 & 5.085 & 7 & 7.422 & -2.357 & -0.008 & 5.056 \\
\hline 0.02609 & 9.501 & -4.420 & -0.028 & 5.053 & 8 & 7.422 & -2.362 & -0.003 & 5.056 \\
\hline 0.03913 & 8.285 & -3.221 & -0.008 & 5.056 & 9 & 7.422 & -2.364 & -0.001 & 5.057 \\
\hline \multirow{9}{*}{0.05218} & 7.422 & -2.362 & -0.003 & 5.056 & 10 & 7.422 & -2.365 & -0.000 & 5.056 \\
\hline & & & & & 11 & 7.422 & -2.365 & -0.000 & 5.056 \\
\hline & & & & & 12 & 7.422 & -2.366 & -0.000 & 5.056 \\
\hline & & & & & 13 & 7.422 & -2.367 & -0.000 & 5.055 \\
\hline & & & & \multicolumn{6}{|c|}{$\rho=1.35$ and $R_{\mathrm{SW}}=0.00781$} \\
\hline & & & & & 8 & 13.120 & -2.182 & -0.003 & 10.935 \\
\hline & & & & $\rho$ & $\beta k_{\mathrm{EC}}^{*} / 2$ & $\beta F_{\text {ref }}^{(\mathrm{CM})} / N$ & $\beta / 2 \int_{k_{\mathrm{EC}}^{*}}^{0} d k_{\mathrm{EC}}\left\langle r^{2}\right\rangle$ & $-\ln \langle\langle\ldots\rangle\rangle / N$ & $\beta F_{\text {conf }}^{(\mathrm{CM})} / N$ \\
\hline & & & & 1.05 & 2000 & 9.679 & -4.621 & 0.000 & 5.058 \\
\hline & & & & 1.35 & 60000 & 14.506 & -3.572 & 0.001 & 10.935 \\
\hline
\end{tabular}

presents two extreme cases: close to melting, $\rho \sigma^{3}=1.05$, and near-close packing, $\rho \sigma^{3}=1.35$. To visualize more easily the exponential character of $\langle n\rangle$ at not-too-low values of $\beta \epsilon$, a logarithmic scale is used; note that the exponential behavior is reached for similar values of the coupling parameter. The largest effect of density is on the exact reference contribution, see Table I.

The situation is similar in the FL method. Unfortunately, the behavior of the different contributions is just the opposite. The intrinsic drawback of the FL method comes in the end from the way the coupling parameter of the reference system is chosen. In effect, using the harmonic coupling potential (28)

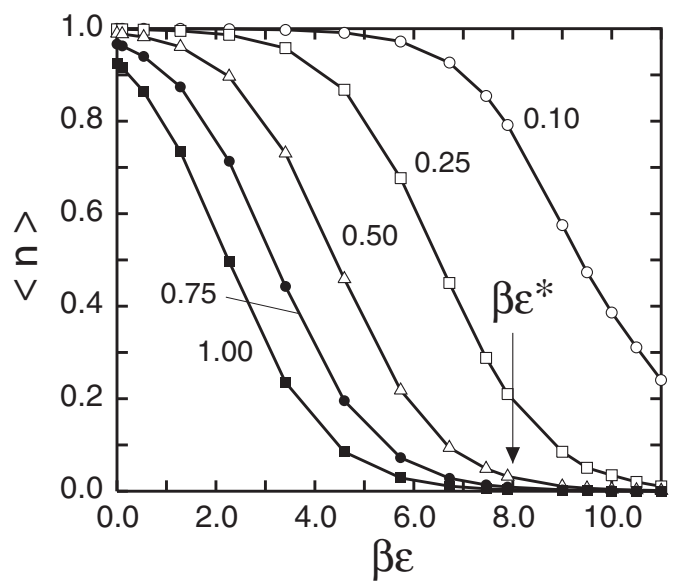

FIG. 1. $\langle n\rangle$ versus $\beta \epsilon$ for the HS model and different values of $R_{\mathrm{SW}}=\gamma R_{\max }$ (with $\left.R_{\max }=0.05218 \sigma\right)$. The values of $\gamma$ are indicated with labels; from left to right: $\gamma=1,0.75,0.50,0.25$, and 0.10 . The reduced density is $\rho \sigma^{3}=1.05$, the number of particles $N=4000$, and the maximum value of $\beta \epsilon^{*}$ (indicated with an arrow) is $\beta \epsilon^{*}=8$. The solid lines are a guide to the eye. in (29), one obtains

$$
\begin{aligned}
\frac{\beta F_{\mathrm{conf}}^{(\mathrm{CM})}}{N}= & -\frac{1}{N} \ln \left[\left(\frac{2 \pi}{\beta k_{\mathrm{EC}}^{*}}\right)^{3 N / 2} N^{3 / 2}\left(\frac{\beta k_{\mathrm{EC}}^{*}}{2 \pi}\right)^{3 / 2}\right] \\
& +\frac{\beta}{2} \int_{k_{\mathrm{EC}}^{*}}^{0} d k_{\mathrm{EC}}\left\langle r^{2}\right\rangle_{k_{\mathrm{EC}}}-\frac{1}{N} \ln \left\langle\left\langle e^{-\beta U_{\mathrm{HS}}}\right\rangle\right\rangle_{k_{\mathrm{EC}}^{*}} .
\end{aligned}
$$

The first term diverges logarithmically with $k_{\mathrm{EC}}^{*}$ to $+\infty$. The last term, the reference free-energy correction, is a small positive contribution. The coupling term is negative and diverges logarithmically with $k_{\mathrm{EC}}^{*}$ to $-\infty$. To improve the accuracy of the difference between the two divergent contributions, $k_{\mathrm{EC}}^{*}$ should not be too large. However, the

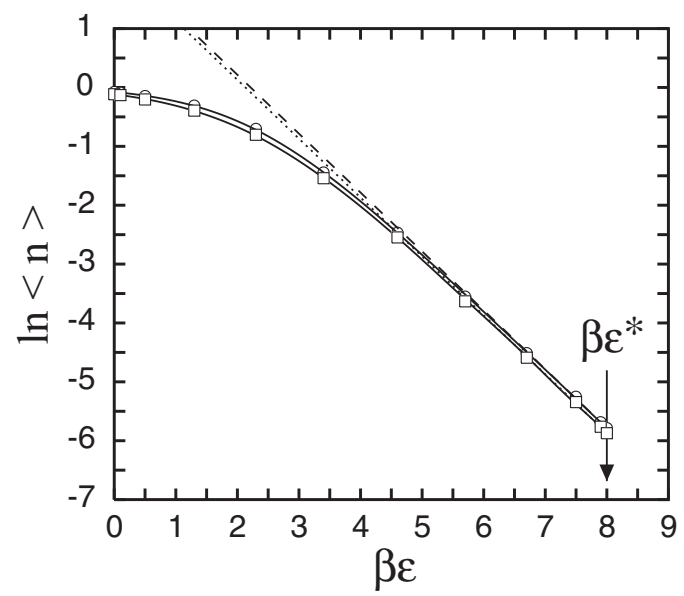

FIG. 2. $\ln \langle n\rangle$ versus $\beta \epsilon$ for the HS model at reduced densities $\rho \sigma^{3}=1.05$ (circles) and 1.35 (squares), with $R_{\mathrm{SW}}=0.05218 \sigma$ and $0.00781 \sigma$, respectively. The number of particles is $N=4000$, and $\beta \epsilon^{*}=8$ (indicated with an arrow). The straight lines are fits for high values of $\beta \epsilon$. The solid lines are a guide to the eye. 


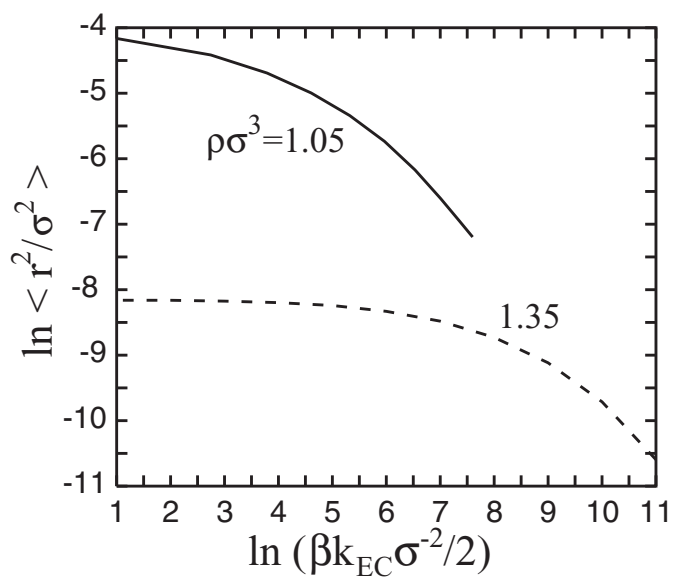

FIG. 3. $\ln \left\langle r^{2} / \sigma^{2}\right\rangle$ versus $\ln \left(\beta k_{\mathrm{EC}} \sigma^{-2} / 2\right)$ for the HS model at reduced densities $\rho \sigma^{3}=1.05$ (continuous curve) and 1.35 (dashed curve). Solid lines are a guide to the eye.

expression for the reference free energy is valid for large $k_{\mathrm{EC}}^{*}$. Consequently, we are forced to take an arbitrarily large value and to evaluate the correction [last term in (32)], which requires an extra simulation or theoretical approximation. The problem becomes worse at high densities, where the parameter $k_{\mathrm{EC}}^{*}$ can be very large.

Finally, the thermodynamic integral (second term) does not show any significant difference with the equivalent term of Eq. (31) except, perhaps, that it is simpler to count particles than to determine the mean-square displacement. Also, the conspicuous cusp presented by $\left\langle r^{2}\right\rangle$ at $k_{\mathrm{EC}}=0$ requires us to perform the thermodynamic integral carefully.

To compare the two approches more quantitatively, we have used the FL method to obtain $\beta F_{\text {conf }}^{(\mathrm{CM})} / N$ at reduced densities $\rho \sigma^{3}=1.05$ and 1.35. As expected, the results agree with those of the SW approach (see Table I). The correction term (32) was calculated with the expression derived by Frenkel and Ladd [1]. Note that for $\rho \sigma^{3}=1.05, \beta k_{\mathrm{EC}}^{*} / 2 \gtrsim 2000$ is a reasonable value which puts the correction term within the required accuracy; however, for $\rho \sigma^{3}=1.35, \beta k_{\mathrm{EC}}^{*} / 2$ increases to 50000 but the correction is not negligible. The problem, in both cases, is that the correction has to be evaluated before assessing if it is negligible or not. Figure 3 shows $\left\langle r^{2}\right\rangle$ as a function of $\beta k_{\mathrm{EC}} / 2$ for the two values of density. Note that, in this density range, $\left\langle r^{2}\right\rangle$ and $\beta k_{\mathrm{EC}} / 2$ change about two orders of magnitude, and a double logarithmic scale is necessary to present the two curves in the same figure. The contrast between Figs. 2 and 3 is very significant.

From the above discussion, it is clear that the use of a SW model leads to a much simpler procedure to obtain the free energy than the harmonic potential. We have presented data for the HS model, but the discussion is similar for other systems, as will be shown elsewhere.

\section{CONCLUSIONS}

In this paper we have developed a general approach to evaluate the absolute free energy of a crystal with constrained center of mass using MC simulation. From a fundamental point of view, we have justified the popular method of Frenkel and Ladd, one of the few available methods to calculate the free energy of a statistical system. In our approach one can choose the most convenient coupling potential for each problem. We have proposed a particular coupling potential, the SW model, which can be used in general and provides a simplified procedure, while giving more accurate results than the standard harmonic potential of the FL method. The choice of a SW model is simpler because it gives a universal procedure, only one coupling parameter is needed, and no extra simulations are required to compute extra corrections to the free energy. Two parameters are needed: the radius $R_{\mathrm{Sw}}$, which is set such that the simple and exact expression of the reference free energy is minimized, and the coupling constant $\epsilon^{*}$, which can be set to $\sim 10 k T$ regardless of the value of density. None of these desirable features are inherent to the FL method. Also, in the FL method there is no criterion to determine the maximum coupling parameter $k_{\mathrm{EC}}^{*}$ other than to take it as large as possible. The problem becomes worse at high densities. The thermodynamic integral is extended over a large interval in the coupling parameter, while the corresponding integral with a SW coupling extends up to a value of $10 \mathrm{kT}$ in all cases. The SW potential gives more accurate results because the reference free energy is exact and the integrand goes to zero exponentially. The accuracy only depends on the MC statistics to obtain the thermodynamic integral. In the FL method the situation is rather different: The free energy of the noninteracting reference system is an approximation, whose accuracy may be high for large $k_{\mathrm{FC}}^{*}$ but in any case unkown. The correction term due to particle interactions in the reference system has only been approximated for the HS model. Everything depends on how large the coupling parameter $k_{\mathrm{EC}}^{*}$ is but, due the logarithmic divergence of the free energy, the accuracy of the FL method is difficult to assess.

\section{ACKNOWLEDGMENTS}

Financial support from MINECO (Spain) under Grant No. FIS2013-47350-C5-1-R is acknowledged.

\section{APPENDIX: GENERAL EXPRESSION FOR A NONINTERACTING CONFIGURATION PARTITION FUNCTION UNDER THE MASS CENTER CONSTRAINT}

For convenience we translate the particle coordinates $\boldsymbol{r}_{i}$ in the noninteracting partition function, Eq. (14), to their respective lattice vectors, $\boldsymbol{r}_{i}+\boldsymbol{R}_{i} \rightarrow \boldsymbol{r}_{i}$. Then:

$$
\begin{aligned}
\delta\left(\boldsymbol{R}-\frac{1}{N} \sum_{i=1}^{N} \boldsymbol{r}_{i}\right) & \rightarrow \delta\left(\boldsymbol{\Delta}-\frac{1}{N} \sum_{i=1}^{N} \boldsymbol{r}_{i}\right) \\
& =N^{3} \delta\left(N \boldsymbol{\Delta}-\sum_{i=1}^{N} \boldsymbol{r}_{i}\right)
\end{aligned}
$$

with $\boldsymbol{\Delta} \equiv \boldsymbol{R}-\boldsymbol{R}_{\mathrm{GC}}$ and $\boldsymbol{R}_{\mathrm{GC}}$ the geometrical center, defined by:

$$
\boldsymbol{R}_{\mathrm{GC}}=\frac{1}{N} \sum_{i=1}^{N} \boldsymbol{R}_{i} .
$$


Therefore the partition function can be expressed by:

$$
\begin{aligned}
Z_{\mathrm{ref}}^{(\mathrm{CM}, 0)}(\boldsymbol{\Delta})= & N^{3} \int_{V} d \boldsymbol{r}_{1} \ldots \int_{V} d \boldsymbol{r}_{N} \delta \\
& \times\left(N \boldsymbol{\Delta}-\sum_{i=1}^{N} \boldsymbol{r}_{i}\right) e^{-\beta \sum_{i=1}^{N} \lambda^{*} V_{i}} .
\end{aligned}
$$

Now we define the effective volume explored by each noninteracting particle, weighted with the Boltzmann factor:

$$
V_{\mathrm{eff}}=\int_{V} d \boldsymbol{r} e^{-\beta \lambda^{*}\left[V(\boldsymbol{r})-V_{0}\right]},
$$

and the probability density $\omega(\boldsymbol{r})$ for a particle to be localized at $r$ :

$$
\omega(\boldsymbol{r})=\frac{1}{V_{\mathrm{eff}}} e^{-\beta \lambda^{*}\left[V(\boldsymbol{r})-V_{0}\right]},
$$

where $V_{0}$ is the minimum of $V(\boldsymbol{r})$. Note that these two definitions are independent of the $\mathrm{CM}$ constraint. The configuration partition function $Z_{\mathrm{ref}}^{(\mathrm{CM}, 0)}(\boldsymbol{\Delta})$ becomes:

$$
\begin{aligned}
Z_{\mathrm{ref}}^{(\mathrm{CM}, 0)}(\boldsymbol{\Delta})= & N^{3} V_{\mathrm{eff}}^{N} e^{-\beta N \lambda^{*} V_{0}} \int_{V} d \boldsymbol{r}_{1} \omega\left(\boldsymbol{r}_{1}\right) \ldots \\
& \times \int_{V} d \boldsymbol{r}_{N} \omega\left(\boldsymbol{r}_{N}\right) \delta\left(N \boldsymbol{\Delta}-\sum_{i=1}^{N} \boldsymbol{r}_{i}\right),
\end{aligned}
$$

which, except for the prefactors, is formally identical to the probability density of a weighted random-walk problem, i.e., the probability density that, after a sequence of $N$ displacements of size $\boldsymbol{r}_{i}$ and probability density $\omega\left(\boldsymbol{r}_{i}\right)$, a given particle arrives at $N \boldsymbol{\Delta}$. In the present context it is the probability density that, after displacements of size $\boldsymbol{r}_{i}$ and probability density $\omega\left(\boldsymbol{r}_{i}\right)$ of the $N$ particles about their lattice sites, the CM has been displaced by $\boldsymbol{\Delta}$ from the geometrical center. Now we follow standard random-walk theory where a system of $N$ particles gives a step, instead of having a single particle giving $N$ steps. Using the Fourier transforms

$$
\delta\left(N \boldsymbol{\Delta}-\sum_{i=1}^{N} \boldsymbol{r}_{i}\right)=\frac{1}{(2 \pi)^{3}} \int d \boldsymbol{k} e^{-i \boldsymbol{k} \cdot\left(N \boldsymbol{\Delta}-\sum_{i=1}^{N} \boldsymbol{r}_{i}\right)}
$$

and

$$
Q(\boldsymbol{k})=\int d \boldsymbol{r} \omega(\boldsymbol{r}) e^{i \boldsymbol{k} \cdot \boldsymbol{r}},
$$

the partition function $Z_{\mathrm{ref}}^{(\mathrm{CM}, 0)}$, Eq. (A6), can be written as

$$
Z_{\text {ref }}^{(\mathrm{CM}, 0)}(\boldsymbol{\Delta})=V_{\mathrm{eff}}^{N} N^{3 / 2} C(N, \boldsymbol{\Delta}),
$$

with

$$
C(N, \boldsymbol{\Delta})=\frac{N^{3 / 2}}{(2 \pi)^{3}} e^{-\beta N \lambda^{*} V_{0}} \int d \boldsymbol{k} e^{-i N \boldsymbol{k} \cdot \boldsymbol{\Delta}} Q^{N}(\boldsymbol{k}) .
$$

For convenience, we have formally written $Z_{\text {ref }}^{(\mathrm{CM}, 0)}$ as the product of three factors; only the third, $C(N, \boldsymbol{\Delta})$, could be, in principle, difficult to evaluate. However, as shown below, it tends very quickly to a constant with $N$, and this constant only depends on the parameters of the coupling potential. If the restriction on the $\mathrm{CM}$ is relaxed, the noninteracting partition function reduces to $Z_{\mathrm{ref}}^{(0)}=V_{\mathrm{eff}}^{N}$, which allows us to interpret the two last factors of Eq. (A9) as a correction to the effective volume due to the $\mathrm{CM}$ constraint.

The presence of the oscillating factor $e^{i k \cdot r}$ in the integrand of Eq. (A8) makes that only low- $k$ values contribute to it. The exponential power $N$ of $Q(\boldsymbol{k})$ enhances extremely this effect in Eq. (A10) so we can develop $Q$ in a $k$ series and, in practice, keep the first nonzero term. Developing $Q$ in a $k$ series:

$$
Q(\boldsymbol{k})=i \boldsymbol{k}\langle\boldsymbol{r}\rangle+\frac{k^{2}}{2}\left\langle\Delta r^{2} \cos ^{2} \theta\right\rangle+O^{3}(k)
$$

with $\left\langle\Delta r^{2} \cos ^{2} \theta\right\rangle \equiv\langle\boldsymbol{r} \cos \theta\rangle^{2}-\left\langle r^{2} \cos ^{2} \theta\right\rangle$ and

$$
\langle\boldsymbol{r} \cos \theta\rangle \equiv \int_{V} d \boldsymbol{r} \omega(\boldsymbol{r}) \boldsymbol{r} \cos \theta
$$

and

$$
\left\langle r^{2} \cos ^{2} \theta\right\rangle \equiv \int_{V} d \boldsymbol{r} \omega(\boldsymbol{r}) r^{2} \cos ^{2} \theta .
$$

Using these results, the partition function reads:

$$
\begin{aligned}
Z_{\mathrm{ref}}^{(\mathrm{CM}, 0)}(\boldsymbol{\Delta})= & V_{\mathrm{eff}}{ }^{N} \frac{N^{3}}{(2 \pi)^{3}} e^{-\beta N \lambda^{*} V_{0}} \\
& \times \int d \boldsymbol{k} e^{-i N \boldsymbol{k} \cdot \boldsymbol{\Delta}} e^{i N \boldsymbol{k} \cdot\left\langle\boldsymbol{r} \cos \theta+\frac{N k^{2}}{2}\left\langle\Delta r^{2} \cos ^{2} \theta\right\rangle+N O^{3}(k)\right.} .
\end{aligned}
$$

When integrated, one obtains an expression in terms of parabolic functions (see Ref. [7]). Equation (A14) is the most general expression for $Z_{\mathrm{ref}}^{(\mathrm{CM}, 0)}$. However, as mentioned in the main text, interesting cases correspond to $\Delta=0$ and $\omega=\omega(|\boldsymbol{r}|)$. For these cases $\langle\boldsymbol{r} \cos \theta\rangle=0$, and Eq. (A14) reduces to

$$
Z_{\mathrm{ref}}^{(\mathrm{CM}, 0)}(0)=\frac{V_{\mathrm{eff}}^{N} N^{3}}{(2 \pi)^{3}} e^{-\beta N \lambda^{*} V_{0}} \int d \boldsymbol{k} e^{-\frac{N k^{2}}{2}\left\langle r^{2} \cos ^{2} \theta\right\rangle} .
$$

Performing the integrals over $\boldsymbol{k}$ and the angular variables, we finally obtain

$$
Z_{\mathrm{ref}}^{(\mathrm{CM}, 0)}(0)=V_{\mathrm{eff}}^{N} N^{3 / 2} e^{-\beta N \lambda^{*} V_{0}}\left[\frac{8}{3} \pi^{2} \int d r r^{4} \omega(r)\right]^{-3 / 2}
$$

[1] D. Frenkel and A. J. C. Ladd, J. Chem. Phys. 81, 3188 (1984).

[2] D. Frenkel and B. Smit, Understanding Molecular Simulation (Academic Press, New York, 2002).

[3] P. A. Monson and D. A. Kofke, Adv. Chem. Phys. 115, 113 (2000).
[4] C. Vega, E. Sanz, J. L. F. Abascal, and E. G. Noya, J. Phys.: Condens. Matter 20, 153101 (2008).

[5] G. Navascués and E. Velasco, J. Chem. Phys. 132, 134106 (2010).

[6] G. Kirkwood, J. Chem. Phys. 3, 300 (1935).

[7] I. S. Gradshteyn and I. M. Ryzhik, Table or Integrals, Series, and Products, 2nd ed. (Academic Press, New York, 1980), p. 337. 